\title{
The association between placenta previa and leukocyte and platelet indices - a case control study
}

\author{
Ali O. Ersoy, Sibel Ozler, Efser Oztas, Ebru Ersoy, Ayse Kirbas, Nuri Danisman \\ Zekai Tahir Burak Women's Health Care, Training and Research Hospital
}

\begin{abstract}
Objectives: Despite medical advances, rising awareness, and satisfactory care facilities, placenta previa (PP) remains a challenging clinical entity due to the risk of excessive obstetric hemorrhage. Etiological concerns gave way to life-saving concerns about the prediction of maternal outcomes due to hemorrhage. Our study aimed to detect an early predictive marker of placenta previa.
\end{abstract}

Material and methods: Ninety-three pregnant patients diagnosed with PP and 247 controls were recruited for this retrospective study. Platelet and leukocyte indices were compared between the two groups.

Results: The groups were similar with regard to age distribution (31.2 \pm 5.1 years [mean \pm SD] in the PP group and $31.7 \pm 4.2$ years in controls), body mass index (BMI) $\left(27.7 \pm 3.6 \mathrm{~kg} / \mathrm{m}^{2}\right.$ in the PP group and $27.4 \pm 4.6 \mathrm{~kg} / \mathrm{m}^{2}$ in controls), and most characteristics of the obstetric history. Total leukocyte count, neutrophil count, and neutrophil-to-lymphocyte ratio were significantly higher in the PP group. Mean platelet volume (MPV) and large platelet cell ratio (P-LCR) values were significantly lower in the PP group as compared to controls, with regard to third trimester values. However, patients who were diagnosed postnatally with placenta percreta had lower MPV and P-LCR values than other patients with PP. There were no statistically significant differences between the two groups as far as first trimester values were concerned.

Conclusions: Platelet and leukocyte indices in the third trimester of pregnancy may be valuable predictors of placenta previa and placenta percreta. More comprehensive studies are needed to address this issue.

Key words: prediction, placenta previa, placenta percreta, platelet indices, leukocyte indices

Ginekologia Polska 2016; 87, 5: 367-371

\section{INTRODUCTION}

Placenta previa (PP), defined as a placenta located on the internal os of the uterine cervix, may be the most dangerous cause of excessive obstetric hemorrhage and a life-threatening clinical entity. It sometimes has a storming effect during the delivery as the result of unanticipated catastrophic complications, e.g. excessive hemorrhage, the need for cesarean hysterectomy, or surgical complications (laceration of the urinary bladder, intestinal or vascular trauma). The diagnosis may be delayed due to the fact that PP may cause some mild complications which neither bother the patient nor alarm the clinician until the delivery, except for insignificant intermittent hemorrhage periods [1].

As the time of delivery approaches, questions about the prediction of adhesive placental abnormalities and possible blood loss begin to appear. Ultrasonography and magnetic resonance imaging (MRI) have been proven to be useful in predicting and lessening the complications of placenta previa but, as most screening methods, they are not without pitfalls either [2].

\section{OBJECTIVES}

The literature offers some reports on the prediction of placenta previa but, to the best of our knowledge, leukocyte and platelet indices have never been studied in that context. Therefore, we aimed to detect an early predictive marker of placenta previa and adhesive placental abnormalities using simple laboratory variables.

\section{MATERIAL AND METHODS}

A total of 321 patients diagnosed with placenta previa (PP) and 389 patients diagnosed with low-risk pregnancy, 
admitted to the Zekai Tahir BurakWomen's Health Care Training and Research Hospital in Ankara, Turkey, between January 2011 and December 2013 for delivery, were analyzed retrospectively in this case-control study. The diagnoses and laboratory results were obtained from patient medical records. The diagnosis of PP was made if the lower edge of placental tissue was within $20 \mathrm{~mm}$ of the internal cervical os, or it overlapped it on transvaginal ultrasonographic examination. Placenta percreta was diagnosed when the placenta has invaded the myometrium and the perimetrium. The final pathologic diagnosis of placenta percreta was confirmed after delivery for every subject. The inclusion criteria were as follows: white Turkish female, history of one cesarean section, singleton pregnancy with live fetus $>26$ weeks of gestation (gestational age was determined from the date of the last menstrual period and confirmed by means of ultrasound in the first trimester). Exclusion criteria were as follows: twin gestation, infectious or hemorrhagic problems in the current pregnancy, neonate with congenital anomaly, prior two or more cesarean deliveries, systemic diseases (e.g. hypertensive, diabetic, cardiovascular, renal, thyroidal diseases, infectious diseases, diseases interfering with leukocyte counts and functions), patients who had been using various drugs (painkillers, alcohol, cocaine), and smoking. In the end, 93 patients diagnosed with placenta previa (study group) and 247 pregnant patients (control group) were included in the study after the exclusion criteria had been applied. Local Ethics Committee approved of the study (no.: 27.05.2013/29).

Hematologic parameters in the first and third trimester, demographic data (maternal age, body weight and height, gestational age at birth, birthweight (BW), gender of the neonate and previous reproductive history of the patients) were obtained from their medical records. All of the patients delivered via cesarean section due to PP or prior cesarean section, so the mode of delivery was not included in the analysis. Normal C-Reactive Protein (CRP) levels as well as negative cervical and urinary culture results indicated that the high leukocyte counts were irrespective of any infectious process. BMI was defined as the weight in kilograms divided by the square of the height in meters $\left(\mathrm{kg} / \mathrm{m}^{2}\right)$.

Hematologic parameters were analyzed with $\mathrm{LH} 780$ hematological analyzer (Beckman Coulter, Fullerton, CA, USA). All blood analyses were performed within two hours of blood sampling. Neutrophil-to-lymphocyte ratio (NLR) was calculated by proportioning absolute neutrophil count to absolute lymphocyte count. Likewise, platelet-to-lymphocyte ratio (PLR) was calculated as the ratio between the absolute platelet count and absolute lymphocyte count.

Patient demographic data and hematologic parameters were compared. Statistical Package for Social Sciences (SPSS, Chicago, IL, USA) version 17.0 for Windows was used for statistical analysis. Distribution of the data was analyzed with the Kolmogorov-Smirnov and the Shapiro Wilk test. Data were presented as mean with standard deviation or median with minimum and maximum values of ranges for continuous variables, and as number with percentage for categorical variables. Independent samples t-test was used for parametric variables and Mann-Whitney $U$ test was used for non-parametric variables to compare the investigated groups. Proportions were compared with the Chi-square test or Fisher's exact test. In all analyses, two-tailed $p$-values of $<0.05$ were considered as statistically significant.

\section{RESULTS}

The two groups were similar with regard to age distribution $(31.2 \pm 5.1$ years [mean $\pm \mathrm{SD}$ ] for the PP group and $31.7 \pm 4.2$ years for controls), BMI $\left(27.7 \pm 3.6 \mathrm{~kg} / \mathrm{m}^{2}\right.$ for the PP group and $27.4 \pm 4.6 \mathrm{~kg} / \mathrm{m}^{2}$ for controls), and obstetric history (except number of abortions and curettage) (Table 1). All study participants underwent a cesarean section. Gestational age at birth $(35.7 \pm 3.3$ weeks in the PP group and $38.6 \pm 1.3$ weeks in controls) and birthweight $(2677.9 \pm 744.2$ grams in the PP group and $3395.4 \pm 385.2$ grams in controls) were significantly lower in the PP group as compared to the control group ( $p<0.001 \mathrm{for}$ both groups) (Table 1). No neonatal deaths were recorded.

All hematologic variables were statistically significantly different between the first and the third trimester, except for PLR (Table 2).

Total leukocyte counts, neutrophil counts and neutrophil-to-lymphocyte ratios (NLRs) in the third trimester were significantly higher in the PP group as compared to controls ( $p=0.034, p=0.002$ and $p=0.004$, respectively) (Table 3 ). Mean platelet volume (MPV) and the 'ratio of large platelets' (P-LCR) values in the third trimester were significantly lower in the PP group as compared to controls $(p=0.001$ and $p=0.003$, respectively). There were no differences between groups in terms of lymphocyte counts, platelet counts, 'plateletcrit' (PCT) and 'platelet distribution width' (PDW) values in the third trimester and all of the investigated parameters in the first trimester.

Six out of 93 patients underwent hysterectomy because of adhesive placental abnormalities and all of the 6 patients were diagnosed with total placenta previa. The final pathologic diagnosis of placenta percreta was confirmed after delivery for every subject. None of them experienced any peri-operative or post-operative complications. The first trimester whole blood count of these 6 patients could not be obtained from the medical records, so analyses for their hematologic variables were performed with their third trimester values. Gestational age at birth time and birth weights of these six patients were significantly lower than the values of all other participants $(p=0.047$ and 
Table 1. Comparisons of some demographic and descriptive characteristics between two groups

\begin{tabular}{|l|c|c|c|}
\hline Characteristics & Placenta previa $(\boldsymbol{n = 9 3 )}$ & Control $(\boldsymbol{n}=\mathbf{2 4 7})$ & $\boldsymbol{p}$ value \\
\hline Age (years) & $31.2 \pm 5.1(18-42)$ & $31.7 \pm 4.2(26-42)$ & 0.33 \\
\hline BMI $\left[\mathrm{kg} / \mathrm{m}^{2}\right]$ & $27.7 \pm 3.6(19.95-36.7)$ & $27.4 \pm 4.6(16.73-41.09)$ & 0.21 \\
\hline Gravidity & $3(1-10)$ & $2(1-9)$ & 0.442 \\
\hline Parity & $1(0-8)$ & $1(0-6)$ & 0.352 \\
\hline Living child & $1(0-8)$ & $1(0-6)$ & 0.804 \\
\hline Abortus & $0(0-2)$ & $0(0-3)$ & $<0.001$ \\
\hline D \& C & $0(0-5)$ & $0(0-5)$ & 0.016 \\
\hline Gestational ages at birth (weeks) & $35.7 \pm 3.3(26.14-40.28)$ & $38.6 \pm 1.3(35-42)$ & $<0.001$ \\
\hline Birthweight [g] & $2677.9 \pm 744.2$ & $3395.4 \pm 385.2$ & $<0.001$ \\
\hline
\end{tabular}

Results are presented as mean \pm standard deviation (range) or median (range). BMI — body mass index; D \& C - dilatation and curettage

Table 2. Comparisons of leukocytic and thrombocytic indices in all patients between first and third trimester of pregnancy

\begin{tabular}{|l|c|c|c|}
\hline Characteristics & First trimester & Third trimester & $p$ value \\
\hline Total leukocyte count & $8984.74 \pm 2093.16$ & $10260.58 \pm 2317.12$ & $<0.001$ \\
\hline Neutrophil count & $6217.40 \pm 1740.35$ & $7534.10 \pm 2109.70$ & $<0.001$ \\
\hline Lymphocyte count & $2020.50 \pm 601.62$ & $1932.40 \pm 568.07$ & 0.01 \\
\hline Platelet count (× 109/L) & $244.61 \pm 63.87$ & $225.87 \pm 63.13$ & $<0.001$ \\
\hline Mean platelet volume (MPV) (femtoliter) & $10.54 \pm 1.01$ & $10.73 \pm 1.02$ & $<0.001$ \\
\hline Platelet-crit (PCT) & $0.25 \pm 0.06$ & $0.24 \pm 0.06$ & $<0.001$ \\
\hline Platelet distribution width (PDW) (\%) & $12.93 \pm 2.28$ & $13.74 \pm 2.59$ & $<0.001$ \\
\hline Ratio of large platelets (P-LCR) (\%) & $29.83 \pm 7.70$ & $30.81 \pm 7.49$ & 0.016 \\
\hline Neutrophil to lymphocyte ratios (NLR) & $3.30 \pm 1.36$ & $4.32 \pm 2.40$ & $<0.001$ \\
\hline Platelet to lymphocyte ratios (PLR) & $128.73 \pm 42.0$ & $126.17 \pm 51.87$ & 0.376 \\
\hline
\end{tabular}

The data were given as mean \pm standard deviation

Table 3. Comparisons of some haematologic parameters between two groups

\begin{tabular}{|l|c|c|c|}
\hline Characteristics & Placenta previa $(\boldsymbol{n = 9 3 )}$ & Control $(\boldsymbol{n}=\mathbf{2 4 7})$ & $p$ value \\
\hline WBC1 & $\begin{array}{c}8701.11 \pm 2369.21 \\
(4550-15070)\end{array}$ & $\begin{array}{c}9015.75 \pm 2063.77 \\
(4300-14670)\end{array}$ & 0.459 \\
\hline WBC3 & $\begin{array}{c}10834.73 \pm 2591.77 \\
(5920-20490)\end{array}$ & $\begin{array}{c}10188.02 \pm 2145.60 \\
(5540-16300)\end{array}$ & 0.034 \\
\hline Neutrophil count in the $1^{\text {st }}$ trimester & $5.94 \pm 1.81(3.02-10.59)$ & $6.25 \pm 1.73(2.40-11.6)$ & 0.387 \\
\hline Neutrophil count in the $3^{\text {rd }}$ trimester & $8.21 \pm 2.42(3.68-18.43)$ & $7.44 \pm 1.89(3.71-13.3)$ & 0.002 \\
\hline WBC3/WBC1 ratio & $1.28 \pm 0.32(0.76-1.96)$ & $1.17 \pm 0.31(0.54-2.55)$ & 0.090 \\
\hline NLR in the $1^{\text {st }}$ trimester & $3.20 \pm 1.04(1.58-6.00)$ & $3.31 \pm 1.39(1.1-13.56)$ & 0.998 \\
\hline NLR in the $3^{\text {rd }}$ trimester & $5.24 \pm 3.55(2.01-20.94)$ & $4.11 \pm 1.71(1.83-14.54)$ & 0.003 \\
\hline MPV in the ${ }^{15 t}$ trimester & $10.48 \pm 1.17(8.7-13.9)$ & $10.54 \pm 0.99(7.4-13.1)$ & 0.769 \\
\hline MPV in the $3^{\text {rd }}$ trimester & $10.35 \pm 1.02(8.3-12.8)$ & $10.75 \pm 1.01(8.3-13.8)$ & 0.001 \\
\hline P-LCR in the $1^{\text {st }}$ trimester & $29.13 \pm 9.5(15.4-55.9)$ & $29.9 \pm 7.5(3.64-49.9)$ & 0.625 \\
\hline P-LCR in the $3^{\text {rd }}$ trimester & $28.20 \pm 7.44(15.6-46)$ & $30.91 \pm 7.42(13-53.4)$ & 0.003 \\
\hline
\end{tabular}

The data were given as the mean \pm standard deviation (range). WBC1 — total leukocyte count in the first trimester; WBC3 — total leukocyte count in the third trimester; NLR - neutrophil to lymphocyte ratio; MPV — mean platelet volume; P-LCR — ratio of large platelets 
Table 4. Comparisons of characteristics between patients with and without placenta percreta in the group of placenta previa

\begin{tabular}{|l|c|c|}
\hline Characteristics & Placenta percreta $(\boldsymbol{n}=\mathbf{6})$ & Non-percreta placenta previa $(\boldsymbol{n}=\mathbf{8 7})$ \\
\hline Age (years) & $31.67 \pm 4.23$ & $31.27 \pm 5.05$ \\
\hline BMI $\left[\mathrm{kg} / \mathrm{m}^{2}\right]$ & $28.90 \pm 3.14$ & $27.70 \pm 3.62$ \\
\hline Gestational ages at birth (weeks) & $33.16 \pm 4.47$ & $35.93 \pm 3.12$ \\
\hline Birthweight [g] & $2130.0 \pm 955.15$ & $2720.57 \pm 715.58$ \\
\hline WBC3 & $10925.0 \pm 2984.89$ & $10817.27 \pm 2479.48$ \\
\hline Neutrophil count in the $3^{\text {rd }}$ trimester & $8655.0 \pm 3010.94$ & $8140.90 \pm 2275.57$ \\
\hline NLR in the $3^{\text {rd }}$ trimester & $5455.0 \pm 2110.57$ & $5161.9 \pm 3608.57$ \\
\hline MPV in the $3^{\text {rd }}$ trimester & $9.23 \pm 0.59$ & $10.48 \pm 0.98$ \\
\hline P-LCR in the $3^{\text {rd }}$ trimester & $20.65 \pm 3.93$ & 0.604 \\
\hline
\end{tabular}

The data were given as mean \pm standard deviation. $\mathrm{BMI}$ - body mass index; WBC3 - total leukocyte count in the third trimester; NLR - neutrophil to lymphocyte ratio; MPV — mean platelet volume; P-LCR — ratio of large platelets

$\mathrm{p}=0.036$, respectively). Gestational age at birth time of these six patients was significantly lower than values of other patients in the PP group $(p=0.046)$. Birth weights of neonates born to these 6 patients were lower, but without statistical significance, as compared to the others in the PP group ( $p=0.061$ ) (Table 4).

Third trimester MPV values of the patients with placenta percreta were significantly lower than other patients in the PP group ( $p=0.003$ ), but also lower than all other patients included in the study $(p=0.001)$. Likewise, third trimester P-LCR values of these patients were significantly lower than other patients in the PP group ( $p=0.002)$, but also lower than all other subjects included in the study $(p=0.001)$.

\section{DISCUSSION}

Similarity of demographic features such as age, BMI, and most obstetric history characteristics between the PP group and the control group increased the reliability and validity of the comparison. Previous curettage and abortion are widely known risk factors for the development of PP and our findings also confirmed that fact [1, 3]. Birth weight and gestational age at birth in the PP group were significantly lower as compared to controls. We attributed these results to the complicated pregnancies, especially hemorrhagic consequences which demand early delivery. Regardless, no neonatal deaths were noted. It may have been due to low-risk study samples, because we excluded patients with systemic diseases, history of two or more cesarean deliveries, prior infectious or hemorrhagic problems in the current pregnancy and other risk factors in order to provide an equal evaluation between the two groups at the very beginning of the study.

Considerable diversity of the hematologic parameters between the first and the third trimester made us think that pregnancy is a continuously changing process in itself. One study reported that the upper limit of leukocyte count increases from the first to the third trimester, which is con- sistent with our findings [4]. Research about altered plasma volume and cellular content revealed that the increase in the WBC count was largely due to increases in circulating segmented neutrophils and granulocytes, whose absolute number was nearly doubled at term. It was said that the reason for the increased leukocytosis was still unclear, but it might have been caused by the elevated estrogen and cortisol levels [5]. In our study, platelet (PLT) counts of all patients in the first trimester were significantly higher than in the third trimester, which is again consistent with the literature. Pitkin and Witte [6] and O'Brien [7] showed that platelet counts were decreasing with advancing gestational age. In our study, MPV and P-LCR values of all patients in the third trimester were significantly higher than in the first trimester. Diminution of platelet counts and simultaneous enhancement of their volume and distribution width over the third trimester might be caused by their destruction, regarding microangiopathic reasons, even in a normal pregnancy. It has been well-established in a study by Fay et al. [8].

Total leukocyte count, neutrophil count, neutrophil-to-lymphocyte ratio (NLR), MPV and P-LCR values in the third trimester were good predictors for the diagnosis of PP in our study. Other parameters like lymphocyte count, platelet count, PCT, and PDW values in the third trimester and all of the investigated hematologic parameters in the first trimester did not predict placenta previa. Neutrophil dominance in the PP group prompted us to think that an inflammatory process may take place in the third trimester because of PP. It could be caused by an ascending intrauterine and placental infection as a result of close proximity to the cervico-vaginal microbial colonization. It was well-established in a study by Park et al. [9]. Interestingly, there was no difference between patients with placenta percreta and patients with non-percreta placenta previa regarding neutrophil dominance in our study. Considering similar close proximity of the placenta to the uterine cervix in 
these two groups, we can advocate the accuracy of the idea put forward by Park et al. [9]. However, we excluded patients with positive cervical culture result at the beginning of the study. Perhaps inflammatory processes in cervical tissue of the patients with placenta previa were non-infectious or infectious without bacterial colonization [10].

As it was repeatedly confirmed by previous studies, there is no ideal method to predict excessive hemorrhage in patients with placenta previa during the antepartum period because hemorrhage volume is known to be multifactorial, including PP-associated complications. The most common type of complications are adhesive placental disorders, especially placenta percreta. The sensitivity of ultrasound reached $90 \%$ to predict adhesive placental disorders, but there is still lack of evidence to predict the hemorrhage volume [11]. In addition, surgical complications significantly contribute to higher incidence of morbidity to baseline morbidity of placenta previa.

In our study, all six patients with placenta percreta had significantly lower MPV and lower P-LCR values as compared to cases without placenta percreta (non-percreta placenta previa) in the third trimester. Alas, the literature sources offered no explanation for this finding.

Our study was not without limitations. We were not able to access the first trimester hematologic variables of the six patients who were diagnosed with placenta percreta due to the fact that they were referred to our tertiary care hospital from another institution. Another limitation was lack of evidence about the need for blood product transfusion and hemorrhage volume, which was caused by incomplete medical files.

\section{CONCLUSIONS}

MPV and P-LCR values were found to be significantly lower in patients with placenta percreta than in patients with placenta previa non-percreta, and also were significantly decreased in all PP patients as compared to controls. These two parameters seem promising in distinguishing patients with adhesive placental complications from patients who have non-complicated placenta previa. Physio-pathologic mechanisms regarding these variabilities remain to be fully elucidated.

\section{ACKNOWLEDGMENTS}

The results of this study were presented as a poster at the $13^{\text {th }}$ World Congress of Fetal Medicine Foundation (FMF), 2014 ( $28^{\text {th }}$ June- $3^{\text {rd }}$ July), Nice, France.

\section{Conflict of interest}

The authors report no conflict of interest.

\section{REFERENCES}

1. Asicioglu O, Sahbaz A, Gungorduk K, [et al.]. Maternal and perinatal outcomes in women with placenta praevia and accreta in teaching hospitals in Western Turkey. J Obstet Gynaecol. 2014, 34, 462-466.

2. Maher MA, Abdelaziz A, Bazeed MF. Diagnostic accuracy of ultrasound and MRI in the prenatal diagnosis of placenta accreta. Acta Obstet Gynecol Scand. 2013, 92, 1017-1022.

3. Kiondo P, Wandabwa J, Doyle P. Risk factors for placenta praevia presenting with severe vaginal bleeding in Mulago hospital, Kampala, Uganda. Afr Health Sci. 2008, 8, 44-49.

4. Abbassi-Ghanavati M, Greer LG, Cunningham FG. Pregnancy and laboratory studies: a reference table for clinicians. Obstet Gynecol. 2009, $114,1326-1331$.

5. Gordon MC. Maternal Physiology. In: Gabbe SG, Niebyl JR, Simpson JL, Landon MB, Galan HL, Jauniaux ERM, [et al.]. Obstetrics: Normal and Problem Pregnancies, Sixth edition. Philadelphia. Saunders, an imprint of Elsevier Inc. 2012, 53.

6. Pitkin $\mathrm{R}$, Witte $\mathrm{D}$. Platelet and leukocyte counts in pregnancy. JAMA. 1979, 242, 2696.

7. O'Brien JR. Platelet count in normal pregnancy. J Clin Pathol. 1976, 29, 174.

8. Fay RA, Hughes AO, Farron NT. Platelets in pregnancy: hyperdestruction in pregnancy. Obstet Gynecol. 1983, 61, 238-240.

9. Park CW, Moon KC, Park JS, [et al.]. The frequency and clinical significance of intra-uterine infection and inflammation in patients with placenta previa and preterm labor and intact membranes. Placenta. 2009, 30, 613-618.

10. Aydogan P, Kahyaoglu S, Saygan S, [et al.]. Does cervical ureaplasma/mycoplasma colonization increase the lower uterine segment bleeding risk during cesarean section among patients with placenta previa? A cross-sectional study. Eur Rev Med Pharmacol Sci. 2014, 18, 2243-2247.

11. Bowman ZS, Eller AG, Kennedy AM, [et al.]. Accuracy of ultrasound for the prediction of placenta accreta. Am J Obstet Gynecol. 2014, 211, 177.e1-7. 